\title{
IDENTITY WITH CONSTANTS IN A CHEVALLEY GROUP OF TYPE $\mathrm{F}_{4}$
}

\author{
V. NESTEROV AND A. STEPANOV
}

\begin{abstract}
N. L. Gordeev proved that a generalized group identity holds in Chevalley groups with multiply laced root systems. It was also shown that a stronger identity is valid for the Chevalley groups of types $\mathrm{B}_{l}$ and $\mathrm{C}_{l}$. In the present paper, it is proved that this strong identity is fulfilled in Chevalley groups of type $\mathrm{F}_{4}$ and fails to be true in Chevalley groups of type $\mathrm{G}_{2}$. The main result of the paper is the last ingredient in the proof of the claim that the lattice of intermediate subgroups between $G\left(\mathrm{~F}_{4}, R\right)$ and $G\left(\mathrm{~F}_{4}, A\right)$ is standard for an arbitrary pair of rings $R \subseteq A$ with 2 invertible.
\end{abstract}

\section{INTRODUCTION}

In the study of the free generation of subgroups in simple algebraic groups, Gordeev [7] showed that the following identity with constants holds in the Chevalley groups of types $\mathrm{B}_{l}$ and $\mathrm{C}_{l}$ :

$$
\left[X_{\gamma}, X_{\gamma}^{h^{g}}\right]=\{1\}
$$

where $X_{\gamma}$ is a long root subgroup, $h$ is a small semisimple element or a small unipotent element (see Definition 11), and $g$ is a variable.

The present paper supplements and refines the results of [7]. Namely, we prove that the above identity is also valid in the Chevalley groups of type $\mathrm{F}_{4}$ and fails in groups of type $\mathrm{G}_{2}$. Note that our proof, based on another idea (which seems to be simpler), can easily be applied to the case of groups of types $\mathrm{B}_{l}$ and $\mathrm{C}_{l}$ as well.

A group identity with constants in a group $G$ is an equation containing elements of $G$ and a variable (we consider only group identities in one variable). We say that an identity holds in $G$ if it turns into equality when the variable is replaced by an arbitrary element of the group $G$.

In the early 1980s, Golubchik and Mikhalev [1] and Tomanov [5] studied generalized group identities with constants and showed that they can be valid only under very strong restrictions on the coefficients. In the same paper, Golubchik and Mikhalev proved that a certain identity is valid in odd-dimensional orthogonal and symplectic groups.

In the late 1990s, Gordeev showed that every identity with constants in a simple algebraic group must contain a nontrivial small element. Therefore, there is no identity with constants in Chevalley groups corresponding to simply laced root systems. On the other hand, Gordeev proved that the following identity with constants holds true in all Chevalley groups corresponding to multiply laced root systems (i.e., $\Phi=\mathrm{B}_{l}, \mathrm{C}_{l}, \mathrm{~F}_{4}$, and $\left.\mathrm{G}_{2}\right)$ :

$$
\left[X_{\gamma},\left[X_{\gamma}, X_{\gamma}^{h^{g}}\right]\right]=\{1\}
$$

2010 Mathematics Subject Classification. Primary 20 G07.

Key words and phrases. Group identity, Chevalley group, multiply laced root system.

The second author was supported by RFBR (grant no. 08-01-00756-a). 
and that the stronger identity (1) is valid for $\Phi=\mathrm{B}_{l}$ and $\mathrm{C}_{l}$.

Recently, the second author of the present paper discovered that identities with constants in Chevalley groups play a key role in the study of the subgroup lattice of these groups. Using Gordeev's result on the lack of identities with constants, Stepanov has proved that the distribution of subgroups between $G(\Phi, R)$ and $G(\Phi, A)$ is "almost never" standard, provided that $\Phi$ is simply laced. More precisely, the distribution can be standard only if $A$ is integral over $R$, or if the Krull dimension of $R$ equals 1 and $A$ is integral over its localization. On the contrary, in [9, it was proved that for not simply laced root systems $\Phi$, identity (1) implies that the distribution of intermediate subgroups between $G(\Phi, R)$ and $G(\Phi, A)$ is always standard, at least if $\frac{1}{2} \in R$.

It seems that identity (2) does not imply the standard distribution of subgroups. Therefore, the presence of identity (1) in Chevalley groups of type $F_{4}$ turns out to be very important (intuitively, it is clear that identity (1) fails for $\Phi=\mathrm{G}_{2}$; a rigorous proof is given at the end of the current article). This question was stated in the survey of Vavilov and the second author; see [2], Problem 27.

Obviously, it suffices to prove an identity over an algebraically closed field. This implies that it remains true over any ring. Indeed, the identity is inherited by subrings and quotient rings, and any ring is a quotient of a polynomial ring, which embeds into an algebraically closed field.

A small semisimple element is defined up to conjugation. Therefore, identity (1) can be stated in the following way: for any long root $\gamma$ and a small semisimple element $h$, the root subgroup $X_{\gamma}^{h}$ commutes with $X_{\gamma}$. However, it is convenient to assume that $h$ belongs to a torus $T$ such that $X_{\gamma}$ is an elementary root subgroup with respect to $T$.

It is well known (see, e.g., [6]) that any two long root subgroups $X$ and $Y$ are simultaneously conjugate to elementary root subgroups $X_{\alpha}$ and $X_{\beta}$. There exist three essentially different configurations of roots $\alpha$ and $\beta$.

1. $\alpha+\beta \notin \Phi \cup\{0\}$. In this case the subgroups are said to be commuting. In fact, $[X, Y]=\{1\}$.

2. $\alpha+\beta \in \Phi$. In this case we say that these subgroups are noncommuting. The commutator formula $[X,[X, Y]]=\{1\}$ is true.

3. $\alpha=-\beta$. In this case the subgroups are opposite. There are no commutator identities.

In this terminology, identity (2) means that the subgroups $X_{\gamma}$ and $X_{\gamma}^{h^{g}}$ are not opposite, whereas identity (1) means that they commute.

The idea of our proof of identity (1) is very simple. Let $\gamma$ be the maximal root. Then, in a Bruhat decomposition of $g$ one can eliminate the root unipotents commuting with $h$ and with $X_{-\gamma}$. It turns out that the remaining roots generate a root system of type $\mathrm{C}_{m}$ where any two nonopposite long root subgroups commute (here $m=3$ for a root system of type $F_{4}$ and $m=2$ for $\mathrm{B}_{l}$ ). Thus, the result for $\mathrm{B}_{l}$ and $F_{4}$ follows from Lemma 2 of 7 .

A straightforward application of identity (1) in a group of type $\mathrm{C}_{l}$ is cumbersome. Although all remaining root unipotents lie in the group of type $\mathrm{C}_{l}$, the element $h$ does not belong to this group. Hence, one must check that the action of $h$ on the root unipotents is the same as the action of some small element of the group of type $\mathrm{C}_{l}$. On the other hand, in this way we could avoid a reference to Gordeev's result. Indeed, identity (1) in the Chevalley group of type $\mathrm{C}_{3}$ (i.e., in the symplectic group of order 6 ) can be easily verified by matrix calculation.

We produce a detailed proof only for the case where $\Phi=\mathrm{F}_{4}$. However, our arguments work for the group of type $\mathrm{B}_{l}$ as well, without substantial changes. At the same time, 
the idea of the present paper seems to be easier than Gordeev's proof of identity (1) for $\Phi=\mathrm{B}_{l}$.

Notation. Let $G$ be an arbitrary group. For elements $x, y \in G$ we denote by $[x, y]=$ $x y x^{-1} y^{-1}$ the commutator of these elements and by $x^{y}=y^{-1} x y$ the element conjugate to $x$ by $y$. For a subgroup $H$ of $G, H^{x}$ denotes the conjugate subgroup. The mutual commutator of subgroups $F, H \leq G$ is denoted by $[F, H]$.

Now, let $G$ be a Chevalley group with a fixed split maximal torus $T$ and the set of positive roots $\Phi^{+}$. We denote by $B$ the standard Borel subgroup, and by $U$ its unipotent radical.

Let $\Phi$ be a root system of type $\mathrm{F}_{4}$. Fixing an ordering on $\Phi$, we denote by $\gamma$ the maximal root corresponding to this ordering, and by $\rho$ the maximal short root. Let $V$ be a 4 -dimensional vector space with a basis $e_{i}, 1 \leq i \leq 4$. Then (see [3]) the root system $\Phi$ has the following realization:

$$
\Phi=\left\{ \pm e_{i}, \pm e_{i} \pm e_{j}, \frac{1}{2}\left( \pm e_{1} \pm e_{2} \pm e_{3} \pm e_{4}\right)\right\}, \quad \rho=e_{1}, \quad \gamma=e_{1}+e_{2} .
$$

\section{$\S 1$. MAin Result}

Let $K$ be a field, and let $G=G(\Phi, K)$ be a Chevalley group of type $\Phi$.

Definition 1. A noncentral semisimple element $h \in G$ is called a small semisimple element if it is annihilated by all long roots, i. e., $\alpha(h)=1$ for all long roots $\alpha$.

If the root system $\Phi$ is simply laced, i. e., all roots have the same length, then there are no small semisimple elements. In the case of $\Phi=\mathrm{B}_{l}, \mathrm{C}_{l}$, or $\mathrm{F}_{4}$ the small semisimple elements have eigenvalues \pm 1 . Therefore, there are no small semisimple elements in characteristic 2 . In this case a small unipotent element plays the role of a small semisimple element in identity (1).

Definition 2. If char $K=2$, then a nontrivial (not necessarily elementary) short root element is called a small unipotent element. An element is said to be small if it is small semisimple or small unipotent.

Note that the centralizer of a small element is sufficiently large. Due to the condition on the characteristic, the centralizer of a small unipotent element contains root subgroups corresponding to all short roots orthogonal to the given one. These elements are said to be "small" because they have small conjugacy classes.

Lemma 1. Let $\Phi=\mathrm{F}_{4}$. Fix a split maximal torus $T$ in the group $G$ and an ordering on the root system. Let $h \in T$ be a small semisimple element, let $\gamma$ be a maximal root, and let

$$
\Delta=\left\{\alpha \in \Phi^{+} \mid \alpha(h) \neq 1, \alpha-\gamma \in \Phi\right\} .
$$

Then $\Delta$ and $\gamma$ generate a root subsystem of type $\mathrm{C}_{3}$.

Proof. Using the standard realization of the root system of type $\mathrm{F}_{4}$, we immediately check that

$$
\Delta=\left\{e_{1}, e_{2}, \frac{1}{2}\left(e_{1}+e_{2}+e_{3}-e_{4}\right), \frac{1}{2}\left(e_{1}+e_{2}-e_{3}+e_{4}\right)\right\}
$$

We denote these roots by $\alpha_{1}, \alpha_{2}, \alpha_{3}$, and $\alpha_{4}$, respectively.

Then, we have $\alpha_{4}=\alpha_{1}+\alpha_{2}-\alpha_{3}$ and $\gamma=\alpha_{1}+\alpha_{2}$. Therefore, the roots $\alpha_{1}-\alpha_{2}, \alpha_{2}$, and $-\alpha_{3}$ form a fundamental system of roots for the root system of type $\mathrm{C}_{3}$. 
Theorem 1. Let $G=G\left(\mathrm{~F}_{4}, K\right)$ be a Chevalley group of type $\mathrm{F}_{4}$ over a field $K$, and let $h$ be a small element. Then for a long root $\gamma$ and an element $g \in G$ we have

$$
\left[X_{\gamma}, X_{\gamma}^{h^{g}}\right]=\{1\} .
$$

Proof. In accordance with the remark in the Introduction, we may assume $K$ to be an algebraically closed field. Fix a maximal torus $T$ such that $X_{\gamma}$ is an elementary root subgroup with respect to $T$ and fix an ordering on the set of roots such that $\gamma$ is a maximal root.

First, we assume that char $K \neq 2$. Since $g$ is an arbitrary element of the group and $h$ is conjugate to an element of $T$, we may assume that $h \in T$. The set of elements satisfying the required identity is Zariski closed, while the big Bruhat cell is dense. Therefore, we may assume that $g$ lies in the big cell, i.e., $g=u w u_{0}$, where $u_{0}, u \in U, w$ normalizes $T$, and its image $\bar{w}$ in the Weyl group maps $\gamma$ to $-\gamma$.

The left-hand side of our identity is conjugate to $Y_{1}=\left[X_{\gamma}^{g^{-1}}, X_{\gamma}^{g^{-1} h}\right]$. Since $U$ commutes with $X_{\gamma}$ and $X_{\gamma}^{w^{-1}}=X_{\bar{w}^{-1}(\gamma)}=X_{-\gamma}$, we have $Y_{1}=\left[X_{-\gamma}^{u^{-1}}, X_{-\gamma}^{u^{-1} h}\right]$.

We put $\Delta_{1}=\left\{\alpha \in \Phi^{+} \mid \alpha-\gamma \notin \Phi\right\}, \Delta_{3}=\left\{\alpha \in \Phi^{+} \mid \alpha(h)=1\right\}$, and $\Delta_{2}=\Phi^{+} \backslash$ $\left(\Delta_{1} \cup \Delta_{3}\right)$ and represent the element $u^{-1}$ in the form $u^{-1}=u_{1} u_{2} u_{3}$, where $u_{i} \in$ $\prod_{\alpha \in \Delta_{i}} X_{\alpha}$ (the product is taken in a fixed order). The existence of this decomposition follows from Lemma 17 in the book [4. Since $u_{1}$ commutes with $X_{-\gamma}$ and $u_{3}$ commutes with $h, Y_{1}$ is conjugate to $Y_{2}=\left[X_{-\gamma}^{u_{2}}, X_{-\gamma}^{u_{2} h}\right]$.

Since the element $h$ commutes with $X_{-\gamma}$, we have $X_{-\gamma}^{u_{2} h}=X_{-\gamma}^{u_{2}^{h}}$, where $u_{2}^{h} \in \prod_{\alpha \in \Delta_{2}} X_{\alpha}$. Hence, by Lemma 1 the long root subgroups $X_{-\gamma}^{u_{2}}$ and $X_{-\gamma}^{u_{2} h}$ are contained in a subgroup of type $\mathrm{C}_{3}$. By Lemma 2 in [7, they are not opposite (also, this can be checked easily by using the vector representation of a group of type $\mathrm{C}_{3}$ ). At the same time, in the group of type $\mathrm{C}_{l}$ any two nonopposite elementary long root subgroups commute. This completes the proof for a field of characteristic distinct from two.

Now, we consider the case of char $K=2$. Replacing $h$ by its conjugate, we may assume that $h=x_{\rho}(\theta)$, where $\theta \in K \backslash\{0\}$ and $\rho$ is the maximal short root. Using the same arguments as before, we obtain the group $Y_{1}$. Since char $K=2$, any unipotent element $x_{\alpha}(\varepsilon), \alpha \in \Phi^{+}$, commutes with $x_{\rho}(\theta)$. Therefore, $Y_{1}$ is conjugate to $Y_{2}=\left[X_{-\gamma}, X_{-\gamma}^{h}\right]$.

The Chevalley commutator formula implies that $X_{-\gamma}^{h} \subseteq X_{-\gamma} X_{-\gamma+\rho} X_{-\gamma+2 \rho}$. Obviously, each root subgroup on the right-hand side of this inclusion commutes with $X_{-\gamma}$. This completes the proof.

Thus, the theorem above together with Gordeev's result [7] implies the following.

Theorem 2. Let $G$ be a Chevalley group of type $\mathrm{B}_{l}, \mathrm{C}_{l}$, or $\mathrm{F}_{4}$ over a field $K$. Suppose that $h$ is a small element. Then, for any elements $x$ and $y$ in a long root subgroup, the following identity with constants is true: $\left[x, y^{h^{g}}\right]=\{1\}$.

\section{§2. Case where $\Phi=\mathrm{G}_{2}$}

We show that in the case of the Chevalley group of type $\mathrm{G}_{2}$, identity (1) fails.

Let $\Phi^{+}=\{\alpha, \beta, \alpha+\beta, 2 \alpha+\beta, 3 \alpha+\beta, 3 \alpha+2 \beta\}$ be the set of positive roots of the root system $\Phi=\mathrm{G}_{2}$. Here $\alpha$ is short, $\beta$ is long, and $\delta=3 \alpha+2 \beta$ is the maximal root.

If char $K \neq 3$, then $h=h_{\beta}(\omega) h_{3 \alpha+\beta}\left(\omega^{2}\right)$ is a small semisimple element in the group of type $\mathrm{G}_{2}$, where $\omega$ is a primitive third root of unity.

Take $g=x_{-2 \alpha-\beta}(\varepsilon)$ and compute the following commutator:

$$
\left[x_{\delta}(\eta), x_{\delta}(\eta)^{h^{g}}\right]=\left[x_{\delta}(\eta), x_{-2 \alpha-\beta}\left(\varepsilon^{\prime}\right) x_{\delta}(\eta) x_{-2 \alpha-\beta}\left(-\varepsilon^{\prime}\right)\right] .
$$


We have $x_{-2 \alpha-\beta}(\varepsilon)=x_{2 \alpha+\beta}\left(-\varepsilon^{-1}\right) w_{2 \alpha+\beta}\left(\varepsilon^{-1}\right) x_{2 \alpha+\beta}\left(-\varepsilon^{-1}\right)$. Hence, the pair of subgroups $X_{\delta}$ and $x_{-2 \alpha-\beta}\left(\varepsilon^{\prime}\right) X_{\delta} x_{-2 \alpha-\beta}\left(\varepsilon^{\prime}\right)^{-1}$ is conjugate to $X_{\delta}$ and $X_{-3 \alpha-\beta}$. But the mutual commutator subgroup $\left[X_{\delta}, X_{-3 \alpha-\beta}\right]$ is equal to $X_{\beta} \neq\{1\}$.

If char $K=3$, then the identity already fails for $g=1$ and $h=x_{-2 \alpha-\beta}(\varepsilon)$.

\section{REFERENCES}

[1] I. Z. Golubchik and A. V. Mikhalev, Generalized group identities in classical groups, Zap. Nauchn. Sem. Leningrad. Otdel. Mat. Inst. Steklov. (LOMI) 114 (1982), 96-119; English transl. in J. Soviet Math. 27 (1984), no. 4. MR0669562 (83k:20047)

[2] N. A. Vavilov and A. V. Stepanov, Overgroups of semisimple groups, Vestnik Samar. Gos. Univ. Estestvennonauchn. Ser. 2008, no. 3, 51-95. (Russian) MR 2473730 (2009k:20113)

[3] N. Bourbaki, Éléments de mathématique. Fasc. 34. Groupes et algèbres de Lie. Ch. 4-6, Actualités Sci. Indust., No. 1337, Hermann, Paris, 1968. MR0240238(39:1590)

[4] R. Steinberg, Lectures on Chevalley groups. Notes prepared by John Faulkner and Robert Wilson, Yale Univ., New Haven, Conn., 1968. MR0466335 (57:6215)

[5] G. M. Tomanov, Generalized group identities in linear groups, Mat. Sb. (N. S.) 123 (165) (1984), no. 1, 35-49; English transl., Math. USSR-Sb. 51 (1985), no. 1, 33-46. MR.0728928 (85m:20072)

[6] N. A. Vavilov, The geometry of long root subgroups in Chevalley groups, Vestnik Leningrad. Univ. Mat. Mekh. Astronom. 1988, vyp. 1, 8-11; English transl., Vestnik Leningrad Univ. Math. 21 (1988), no. 1, 5-10. MR0946454 (90d:20078)

[7] N. L. Gordeev, Freedom in conjugacy classes of simple algebraic groups and identities with constants, Algebra i Analiz 9 (1997), no. 4, 63-78; English transl., St. Petersburg Math. J. 9 (1998), no. 4, 709-723. MR 1604024 (98m:20056)

[8] A. V. Stepanov, Free product subgroups between Chevalley groups $\mathrm{G}(\Phi, F)$ and $\mathrm{G}(\Phi, F[t])$, Preprint: http://alexei.stepanov.spb.ru/papers/FreeProd.pdf, 2007, to be published in J. Algebra.

[9] _ Subring subgroups in Chevalley groups with doubly laced root systems, Preprint: http://alexei.stepanov.spb.ru/papers/positive.pdf, 2009, to be published in J. Algebra.

Baltic State Technical University, 1-St Krasnoarmeiskaya Street 1, St. Petersburg 190005, RUSSIA

E-mail address: vl.nesterov@mail.ru

St. Petersburg Electrotechnical University, Professor Popov Street 5, St. Petersburg 197376, RUSSIA

E-mail address: stepanov239@gmail.com

Received 8/SEP/2008

Translated by THE AUTHORS 